\title{
X-ray Observations of SN 1993J
}

\author{
H.-U. Zimmermann \\ Max-Planck-Institut für extraterrestrische Physik, Postfach 1312, \\ 85471 Garching, Germany; \\ zim@mpe.mpg. de
}

Summary. In April 2001 SN1993J was observed with both the PN and MOS cameras of the XMM-Newton observatory. A 2-component thermal emission model assuming ionization equilibrium provides a good fit to the spectrum in the 0.3 to $11 \mathrm{keV}$ energy band, but fits to shock models show also acceptable results. The development of the X-ray temperatures over the first 8 years after the explosion is discussed in the light of the standard SN model. The long term X-ray lightcurve shows a general decline of the luminosity with $L_{x} \propto t^{-0.30}$.

\section{Introduction}

Results from spectral and lightcurve analysis as derived from X-ray observations - with special emphasis given to a recent XMM-Newton observation during the first 8 years of this SN are presented and discussed. Part of the work has been done in collaboration with Bernd Aschenbach.

From the XMM-Newton observation in April 2001 we used for our analysis data of about $70 \mathrm{ks}$ from the EPIC PN camera, run in small window mode, and of a similar amount from the MOS2 camera run in imaging mode.

\section{XMM-Newton Spectral Results}

Emission lines of highly ionized Mg, Si, S, Ar, Ca and the complex of the Fe lines dominate the SN spectrum (Fig. 1) at higher energies.

Table 1 shows a compilation of spectral fits to the PN camera, the MOS2 camera, and to a combined spectrum. The differences between the PN and the MOS2 spectra point to residual uncertainties in the spectral cross calibration. Time dependencies observed with the MOS2 calibration and the superior statistics of the PN spectrum with its 7515 counts compared to the 2593 counts in the MOS2 spectrum, let us use for the following spectral discussion only the fitting parameters of the PN spectrum.

The best fit over the whole energy band between 0.3 up to about $11 \mathrm{keV}$ was achieved with a 2-temperature equilibrium model with variable abundances, called vmekal in XSPEC. We have also tested different shock models and found that a Sedov model with variable abundances, called vsedov 


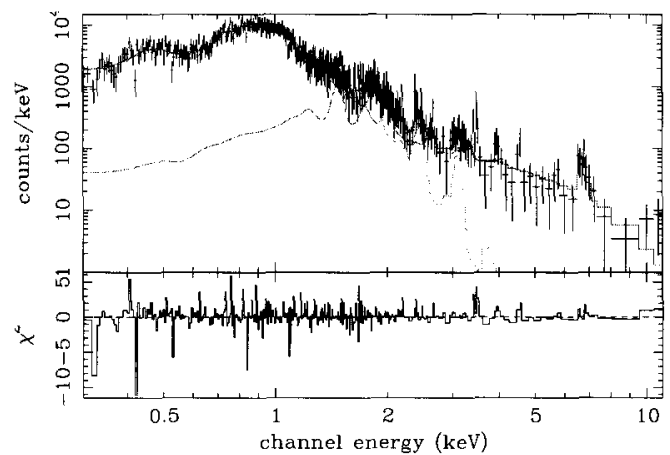

Fig. 1. XMM-Newton spectrum as observed with the PN camera and the best fit thermal 2component model with $k T_{1}=0.34$ $\mathrm{keV}$ and $k T_{2}=6.54 \mathrm{keV}$. The dashed curves show the low and high temperature component.

Table 1. Selected best fit parameters of different spectral models. 2vmekal is a 2component thermal model with variable element abundances, 2vmekall is the same model but with identical element abundances for the 2 temperature components, and vsedov is a shock model with variable abundances.

\begin{tabular}{l|lllllll}
\hline instrument & model & $k T_{1}[k e V]$ & $k T_{2}[\mathrm{keV}]$ & $N_{H 1}\left[10^{22} \mathrm{~cm}^{-2}\right]$ & $N_{H 2}$ & dof & $\chi_{r}^{2}$ \\
\hline PN & 2vmekal & $0.34_{-0.03}^{+0.05}$ & $6.54_{-4}^{+4}$ & $0.55 \pm 0.21$ & $0.33 \pm 1.80$ & 360 & 0.90 \\
PN & 2vmekal1 & $0.35_{-0.04}^{+0.04}$ & $4.24_{-1.8}^{+3.8}$ & $0.45 \pm 0.13$ & $0.18 \pm 0.75$ & 371 & 0.94 \\
PN & vsedov & $0.51_{-0.04}^{+0.04}$ & $7.0_{-0.2}^{+0.2}$ & $0.17 \pm 0.037$ & & 376 & 1.01 \\
MOS2 & 2vmekal & $0.59_{-0.04}^{+0.03}$ & $3.48_{-1.2}^{+1.2}$ & $0.25 \pm 0.25$ & $0.0 \pm 0.54$ & 113 & 1.25 \\
PN+MOS2 & 2vmekal & $0.33_{-0.46}^{+0.46}$ & $4.09_{-1.2}^{+1.2}$ & $0.59 \pm 0.25$ & $0.2 \pm 0.8$ & 505 & 0.99 \\
\hline
\end{tabular}

in XSPEC, produces also acceptable chi-2 values, although all tested shock models reproduce not so well the different line complexes visible in the spectra.

Looking at the abundances of the low temperature component, in the fit $\mathrm{C}, \mathrm{N}$, and $\mathrm{Al}$ are not required, $\mathrm{Mg}$ and $\mathrm{O}$ are under abundant compared to solar, Fe is about solar, and the other elements show clear overabundances.

The high temperature component is determined almost solely by the line complexes of highly ionized $\mathrm{Ar}, \mathrm{Ca}$, and $\mathrm{Fe}$. The temperature of $6.5 \pm 4 \mathrm{keV}$ is poorly restricted, because every temperature change is immediately compensated in the fit by changes in the abundances of these elements. Therefore the element abundances have errors typically much larger than the parameter values themselves. All the elements above Ne, which is not required in the fit, show a clear overabundance, with a moderate value only for the Fe abundance.

\section{Spectral Development}

While the first ROSAT PSPC spectra ([7, 8] reanalyzed for this work [9]) as well as the ASCA measurements [6] did only allow to set lower limits for the 


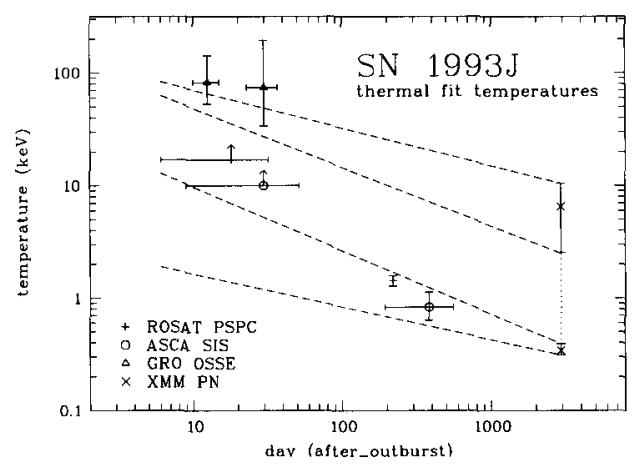

Fig. 2. X-ray determined emission temperatures. The dashed lines outline the time evolution of the low and high temperature component using the standard model. For each component there are two lines bracketing the range of the temperatures allowed by the XMM-Newton data using the 2vmekal model.

temperature of a thermal model, the hard X-ray instrument OSSE on GRO [4] could determine a temperature of approximately $80 \mathrm{keV}$ at these times. ROSAT and ASCA measurements half a year later revealed a strong decrease from the initial high values to temperatures around $1 \mathrm{keV}$ (Fig. 2).

In terms of the standard model proposed by Chevalier [2] it was assumed that the observed emission initially came from the fast forward shock, while emission from the reverse shock region was blocked by absorbing material due to fast cooling processes in the denser environment of this region. For the enormous temperature drop observed it was assumed that the initial absorption disappeared on a time scale of order 100 days so that half a year later the measured flux was dominated by the emission from the reverse shock region.

In the similarity solution of the standard model $[3]$ the ratio between the temperatures in the forward and the reverse shock depend only on $\mathrm{s}$, characterizing the circumstellar density profile and $n$, describing the density profile of the ejecta. Assuming that the two temperature components from the XMM spectrum represent emission from the reverse and forward shock regions and taking the circumstellar density profile $\mathrm{s}=1.65$ derived from the ROSAT light curve (see Section 4 ), we obtain $n=8.9$ for the density profile of the ejecta. We can now enter $\mathbf{n}$ and $\mathrm{s}$ into the expression that describes the temporal development of the temperatures and extrapolate the XMM temperatures backward in time up to the ROSAT, ASCA and GRO results (dashed lines in Fig. 2). Despite the fact that strictly speaking the prior conditions to apply the similarity solution are no more fulfilled at these late times, the temperature development is not too badly described. But we do not think that this can be taken as final proof that we observe X-ray emission from both a forward and a reverse shock region. 


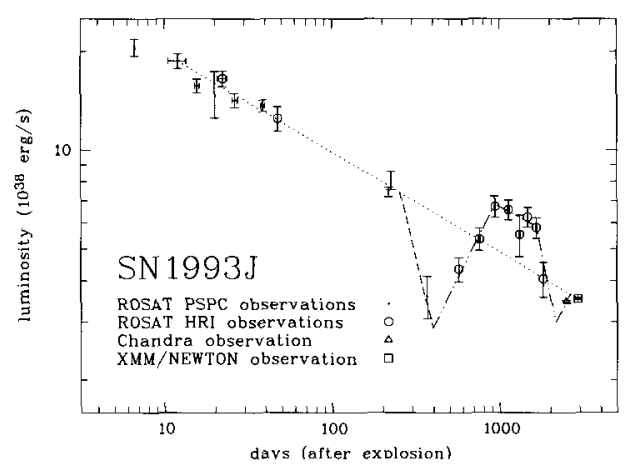

Fig. 3. X-ray light curve showing the development of the X-ray luminosity in the $0.3-2.4 \mathrm{keV}$ energy range. The dotted line indicates a decline rate with $t^{-0.30}$. The dashed lines result from model calculations involving time limited decrease and increase of the density in the ambient matter.

\section{X-ray Lightcurve}

The X-ray lightcurve (Fig. 3), showing the 19 ROSAT observations since day 6, the Chandra observation of 2000 [5] and the XMM observation of 2001, covers now the first 8 years of this SN.

The tendency over the first half year is characterized by a decline of the luminosity with $t^{-0.30}$ where $t$ is the time since the outburst. Thereafter the lightcurve shows a bump and 5 years after the outburst, the luminosity appears to return to the initial decline profile. The Chandra and XMM observations, scaled to the ROSAT data, support the view of a general trend.

In the standard model the circumstellar matter is assumed to originate from the stellar wind of the supergiant progenitor star producing a circumstellar density profile proportional to $r^{-s}$. From the measured time dependence of the luminosity, that is roughly proportional to the square of the density integrated over the emitting volume, we can derive the circumstellar density profile to follow an $r^{-1.65}$ behavior.

In the interpretation that the dominating emission region changed during the first half year from the forward shock to the reverse shock region it is somewhat surprising that the luminosity development does not reflect that dramatic change, but suggests rather a smooth transition maintaining the same gradient. Also the fact that the early ASCA spectrum showed already the Fe complex between 6 and $7 \mathrm{keV}$, demonstrating the existence of material at much lower temperatures than the observed and expected $80 \mathrm{keV}$, shows that reality is certainly more complex.

Dips and bumps in the X-ray lightcurve suggest local density changes above the general power-law profile of the circumstellar matter density, possibly caused by transient changes in the wind parameters of the progenitor or by asymmetries in a possible binary scenario.

If we correlate the ROSAT light curve with the expansion velocity of the SN shell derived from high resolution radio images of this object [1], we notice around the time of the onset of the bump (around day 350) a break in the 
expansion rate. Both features may independently indicate a change of the density profile in the circumstellar medium.

The X-ray lightcurve shows a rapid decline between day 225 and 370 , followed by a rapid increase up to day 935. A similar behavior could have occured between day 1126 and 1464, but there is just one data point consistent with no change. The rapid decline after day 1655 is evident and recovery indicated by the Chandra and XMM points after day 2500. The evidence of the latter 2 dips is statistically not too convincing, but they may be real.

If the shock wave runs into a sufficiently low density regime, matter heated so far will simply expand. For isothermal (adiabatic) expansion the lightcurve will go down with $t^{-2}$ (with $t^{-8 / 3}$, as long as the temperature is higher than about $2 \mathrm{keV}$ ). When thereafter the shock wave encounters a density jump to significantly higher values, the luminosity increases linearly with $t$.

The times when we see after a dip increases of the luminosity in the Xray lightcurve agree surprisingly well with the 3 parts in the radio expansion curve with maximum deceleration. This is interpreted as the moment when the shock hits a step like wall of denser circumstellar material.

We conclude that these changes in the X-ray lightcurve reflect changes of the density in the ambient matter profile, which appear to show some repetitive pattern like in a wave. Whether this characterizes the activity of the progenitor star with respect to mass loss or wind velocity remains to be seen.

\section{Conclusions}

The new data points in the X-ray lightcurve suggest a general decline rate with $L_{x} \propto t^{-0.30}$ with luminosity dips and bumps being intermediate phenomena. The suggested correlation of increases of the X-ray luminosity with the steepest deceleration seen in the expansion of the radio shell and the fact that the circularity of the radio images is not affected by these events, suggests that the observed increases are probably due to density changes in a more or less isotropic circumstellar matter distribution. There is, although statistically weak, an indication of further dips in the X-ray lightcurve, which could be due to repetitive activity changes in the progenitors wind history.

The XMM spectrum is best fitted by a 2-component thermal model that assumes ionization equilibrium. Although the standard model predicts the temperature development not too badly, even at these late times, there remains the question whether in X-rays we really see emission from two locally distinct regions, produced by a forward and a reverse shock.

\section{References}

1. N. Bartel et al. : Astrophys. J. 581, 404 (2002) 
2. R.A. Chevalier: Astrophys. J. 259, 302 (1982)

3. C. Fransson, P. Lundqvist, R.A. Chevalier: Astrophys. J. 461, 993 (1996)

4. M.D. Leising et al. : Astrophys. J. Lett. 431, L95 (1994)

5. D.A. Swartz et al. : Astrophys. J. Suppl. 114, 213 (2003)

6. S. Uno et al. : Astrophys. J. 565, 419 (2002)

7. H.-U. Zimmermann et al. : Nature 367, 621 (1994)

8. H.-U. Zimmermann, W.H.G. Lewin, B. Aschenbach: In: Röntgenstrahlung from the Universe. eds. H.-U. Zimmermann, J. Trümper, H. Yorke (MPE Report) $\mathbf{2 6 3} 289$ (1996)

9. H.-U. Zimmermann, B. Aschenbach: Astron. Astrophys. 406, 969 (2003) 